Document downloaded from:

http://hdl.handle.net/10251/147679

This paper must be cited as:

Clement, J.; Hervás-Peralta, J.; Madrigal-Madrigal, J.; Maestre, H.; Torregrosa, G.; Fernandez-Pousa, CR.; Sales Maicas, S. (2018). Fast Incoherent OFDR Interrogation of FBG Arrays Using Sparse Radio Frequency Responses. Journal of Lightwave Technology. 36(19):4393-4400. https://doi.org/10.1109/JLT.2018.2821199

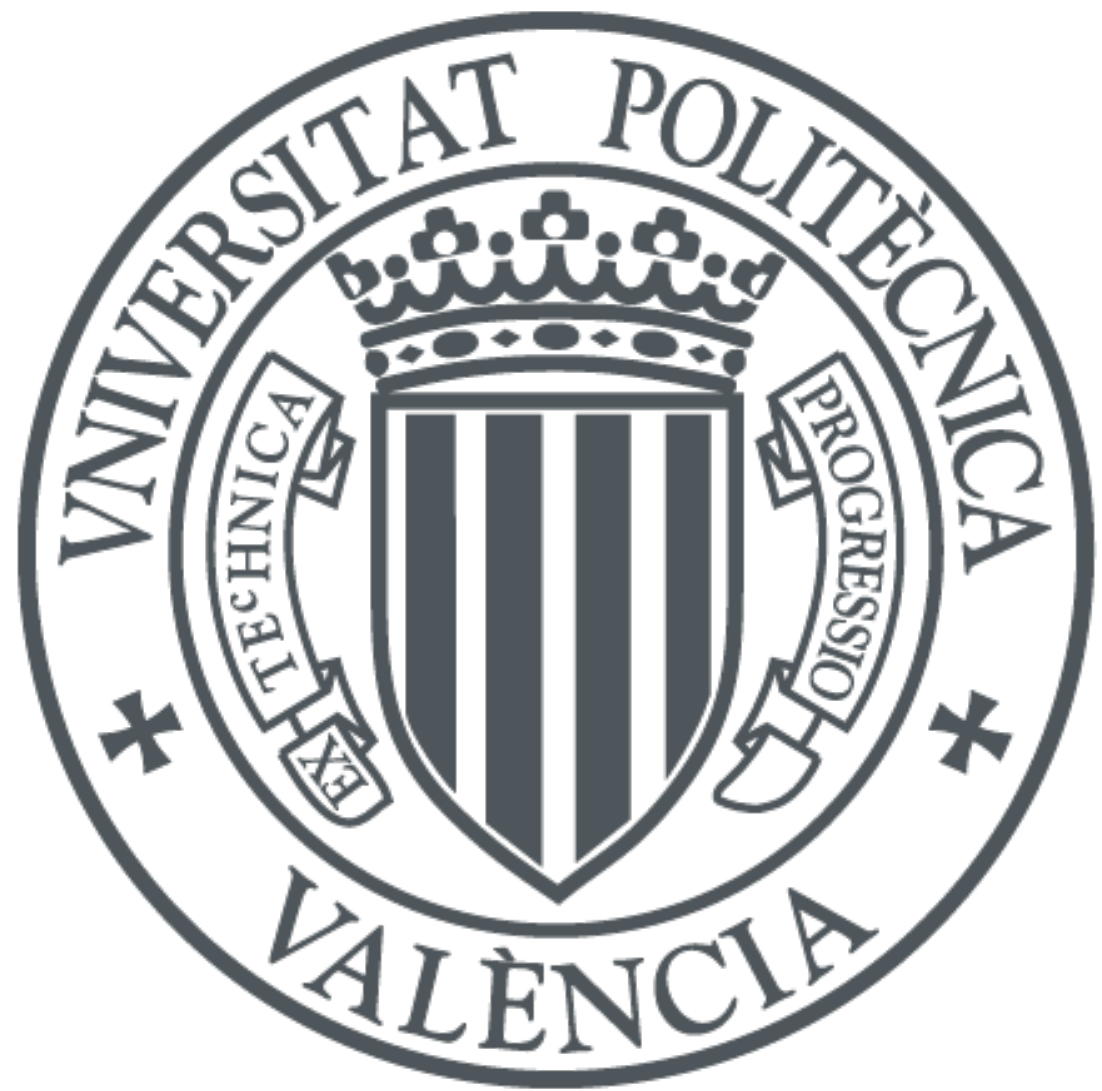

The final publication is available at

https://doi.org/10.1109/JLT.2018.2821199

Copyright Institute of Electrical and Electronics Engineers

Additional Information 


\title{
Fast Incoherent OFDR Interrogation of FBG Arrays Using Sparse Radio-Frequency Responses
}

\author{
Juan Clement, Javier Hervás, Javier Madrigal, Haroldo Maestre, Germán Torregrosa, Member, IEEE, \\ Carlos R. Fernández-Pousa, Senior Member OSA, IEEE, and Salvador Sales, Senior Member OSA, IEEE
}

\begin{abstract}
We present two implementations of fast, discrete incoherent optical frequency-domain reflectometers (I-OFDR) for the interrogation of equally-spaced fiber Bragg grating (FBG) arrays, based on the determination of the array's radiofrequency (RF) response at a sparse number of frequencies. FBG reflectivities are determined by use of the inverse discrete Fourier transform (IDFT) of the sparse RF response, in a dynamic range limited by crosstalk induced by FBG positioning errors. The first implementation employs the complete, vector $\mathrm{RF}$ response at a number of frequencies equal to the number $N$ of FBGs in the array. In the second, the introduction of a reference reflector allows for an interrogation using the power (phaseless) $R F$ response in $4 N-1$ frequencies. Demodulation based on IDFT leads to total interrogation times determined by the network analyzer scan time, which can be as low as $10 \mu \mathrm{s}$ per FBG. Depending on the interrogation technique, electrical bandwidth requirements are 1-2 GHz in our array with 10cm separation. We implemented both techniques in a $N=10$ array, inducing decays in reflectivity by $10 \mathrm{~dB}$ in one or several FBGs. Unambiguous detection of FBG decays was obtained in both interrogation methods. Additional tests performed on the measured reflectivities also show that measurement linearity is preserved in the 10-dB decay range. As discrete I-OFDR systems, the proposed techniques show the possibility to reach compromises between interrogation time and dynamic range or accuracy in reflectivity measurements, using the number of interrogation frequencies and the sensor topology.
\end{abstract}

Index Terms-Optical fiber sensors, fiber Bragg gratings, sensor interrogation, microwave photonics, incoherent OFDR.

\section{INTRODUCTION}

Q UASI-DISTRIBUTED sensors based on arrays of fiber Bragg gratings (FBG), at the same or different nominal reflection wavelength, have become an industry standard due to its mass production as draw tower gratings [1], in scales that may reach thousands of reflectors. Interrogators based on Fabry-Pérot filters [2] or wavelength-swept lasers, both in local [3] and remote [4] configurations, are suitable for conventional wavelength-division multiplexed FBG arrays. In turn, when the reflectors share the same wavelength range, the

Juan Clement, Haroldo Maestre, Germán Torregrosa, and Carlos R. Fernández-Pousa are with the Department of Communications Engineering, Univ. Miguel Hernández, Elche, Spain (e-mail: jclement@umh.es; hmaestre@umh.es; gtorregrosa@umh.es; c.pousa@umh.es)

Javier Hervás, Javier Madrigal, and Salvador Sales are with ITEAM Research Institute, Univ. Politècnica de València, Spain (e-mail: jaherpe2@teleco.upv.es; jamadmad@iteam.upv.es; ssales@dcom.upv.es)

This work was supported in part by Infraestructura GVA-FEDER operative program 2007-2013, and by the Spanish MINECO through project TEC201788029-R. The work of Juan Clement Bellido was supported by the GVA VALi+d scholarship ACIF/2016/214, and that of Javier Hervás Peralta by the Spanish MEC scholarship FPU13/04675.

Manuscript received January 31, 2018 interrogators require both spatial and wavelength selectivity. Demonstrated systems include coherent [5], [6] and incoherent optical frequency-domain reflectometers (OFDR) [7][12]; time-division multiplexing techniques [13]-[16]; optical time-domain reflectometry [17]-[19]; and frequency-shifted interferometers [20], [21].

Among them, incoherent OFDR (I-OFDR) systems, based on similar concepts to those underlying microwave photonics (MWP) filters [22], benefit from the stability of the radiofrequency (RF) interference in the optical incoherent regime, and also of the high signal-to-noise ratio (SNR) offered by electrical narrowband detection [23]. On the downside, optical dynamic range is halved with respect to its value in the electrical domain due to the incoherent regime. Practical figures are limited to $\sim 50 \mathrm{~dB}$ [24], and require low values of intermediate frequency bandwidth (IFBW) that lengthen the measurement time [25]. This fact may preclude the use of I-OFDR methods in scenarios requiring fast detection. A number of techniques can be used to reduce the interrogation time. First, of course, is the increase of IFBW [9]. Second, the use of a sparse number of frequencies in the RF characterization [12]. And third, the use of non-parametric demodulation algorithms with low processing latency, which render the total interrogation time essentially equal to that of trace acquisition.

In this paper, we present two implementations of fast, lowbandwidth I-ODFR interrogators where the aforementioned strategies are incorporated. They are based on the observation that in FBG arrays the position of equally-spaced reflectors is known in advance, at least approximately, so that MWP filtering approaches provide the possibility to allocate sparse RF frequencies adapted to this sensor topology. This approach allows for the increase in interrogation speed while keeping the aforementioned general advantages of I-OFDR techniques. In contrast to the parametric approach used in [12], demodulation is based on the inverse discrete Fourier transform (IDFT) of the retrieved sparse RF response, and therefore the interrogators can be viewed as discrete I-OFDR systems. Deviations in the FBG positioning are shown to induce crosstalk among the power reflected by different elements in the array. The dynamic range becomes limited by crosstalk, and not by noise, and so the IFBW can be increased without incurring in additional penalties. Sparsity and IDFT demodulation thus decrease the scan speed at the expense of accuracy and dynamic range of reflectivity measurements. In our experiments we have used this compromise for the fast detection of coarse values of FBG reflectivities. In the first implementation of the concept, we used as input data the complete (vector) RF transfer function. 
In the second, and following related approaches [26], the use of a reference reflector permits the interrogation using the power (phaseless) response. This second scheme provides an advantage in terms of requirements of instrumentation, since only power measurements in the RF domain are involved.

The interrogation methods are explained in Sections II and III, together with the analysis of crosstalk. The experimental system and the array characterization are presented in Section IV. The results are detailed in Section V, and we end in Section VI with our conclusions.

\section{VECTOR INTERROGATION}

The RF transfer function $H(f)$ of an optical network in the form of an equally-spaced FBG array, such as that shown in Fig. 1(a), can be written as a sum over taps weighted by the reflected optical power:

$$
H(f)=\sum_{n=1}^{N} P_{n} \exp [-j 2 \pi f(n-1) \Delta \tau],
$$

where $N$ is the number of FBGs in the array, $P_{n}$ is the optical power reflected on the $n$-th FBG, $\Delta \tau$ is the round-trip time between two consecutive FBGs, and integer $n$, ranging from 1 to $N$, identifies each FBG in the array. In (1) we have normalized the global delay with respect to the first FBG, so that $H(f)$ becomes periodic in frequency with a free spectral range (FSR) of $1 / \Delta \tau$. The distance between FBGs $\Delta l$ and the corresponding round-trip time $\Delta \tau$ are related as $\Delta l=c \Delta \tau /\left(2 n_{\text {eff }}\right)$, with $c$ the speed of light in vacuum and $n_{\text {eff }}$ the fiber's effective refractive index.

Thus, by choosing a set of interrogation frequencies $f_{k}$ given by:

$$
f_{k}=\frac{k}{N} F S R \quad \text { with } k=0,1, \ldots, N-1,
$$

the transfer function, now discretized, becomes:

$$
H[k]=\sum_{n=1}^{N} P_{n} \exp \left[-j 2 \pi \frac{k(n-1)}{N}\right],
$$

which is the discrete Fourier transform (DFT) of the sequence of reflected powers, or discrete-time impulse response, $h[n]=$ $P_{n+1}$ with $n=0, \ldots, N-1{ }^{1}$ This impulse response can be computed through the IDFT of $H[k]$. In practice, the dc term corresponding to $k=0$ in (2) is obtained from the electrical response at the equivalent frequency $f_{N}=F S R$. The electrical bandwidth required by this method is thus $B=F S R=1 / \Delta \tau$, which is inversely proportional to the FBG separation within the array, and corresponds to the frequency sample of highest frequency. For instance, the bandwidth is as low as $100 \mathrm{MHz}$ for arrays of FBGs separated by 1 meter, increasing to $1 \mathrm{GHz}$ at the 10-cm separation used in our experiments. With an available bandwidth of $50 \mathrm{GHz}$, the spacing can be $\sim 2 \mathrm{~mm}$.

The impact of small deviations in the reflector's positions around their ideal, equally-spaced, values can be analyzed as follows. Let us consider a general situation where the $N$

\footnotetext{
${ }^{1}$ Here we follow the standard convention of discrete-time signals where the running index starts from zero.
}

(a)
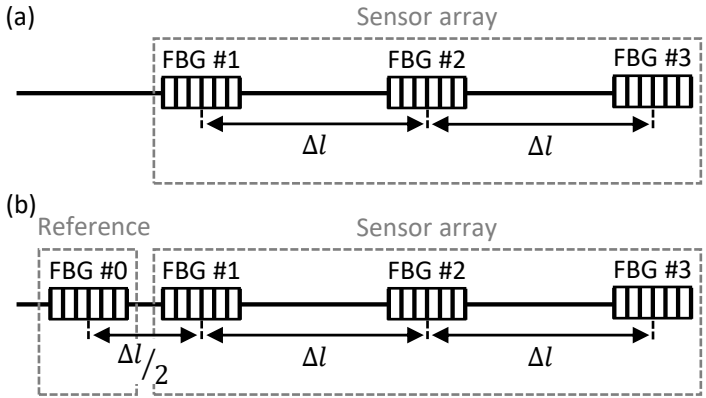

Fig. 1. Scheme of the FBG array composed of three FBGs spaced $\Delta l$ without (a) and with (b) a reference at $\Delta l / 2$.

reflectors are located at positions $\tau_{n}=n \Delta \tau+\delta \tau_{n}$, with $n=0, \ldots, N-1$. The transfer function is:

$$
H(f)=\sum_{n=0}^{N-1} P_{n+1} e^{-j 2 \pi f \tau_{n}}=\sum_{n=0}^{N-1} P_{n+1} e^{-j 2 \pi f \Delta \tau\left(n+u_{n}\right)},
$$

with $u_{n}=\delta \tau_{n} / \Delta \tau$ the relative variation of the $(n+1)$-th reflector position. After discretization and taking the statistical average, denoted with angles, we get:

$$
\begin{aligned}
\langle H[k]\rangle & =\sum_{n=0}^{N-1} P_{n+1} e^{-j 2 \pi k n / N}\left\langle e^{-j 2 \pi k u_{n} / N}\right\rangle \\
& =\Phi(k / N) \sum_{n=0}^{N-1} P_{n+1} e^{-j 2 \pi k n / N}
\end{aligned}
$$

where $\Phi(\nu)$ is the characteristic function of the probability distribution of the relative variations $u_{n}$. These are assumed independent, identically distributed, and Gaussian with standard deviation $\sigma$, so that:

$$
\Phi(\nu)=\langle\exp (-j 2 \pi \nu u)\rangle=\exp \left(-2 \pi^{2} \sigma^{2} \nu^{2}\right) .
$$

Thus, on average, positioning errors amount to low-pass filtering the frequency response. In the reciprocal domain, this low-pass filter mixes the ideal response corresponding to $h[n]=P_{n+1}$ with adjacent sample values, and thus induces crosstalk between reflectors. This (average) crosstalk is, however, of order $\sigma^{2}$, and therefore small, reflecting that the influence of positioning errors is compensated on average.

Of more relevance is the standard deviation of the crosstalk level, which represents an estimate, of order $\sigma$, of the expected impact of a specific distribution of positioning errors $u_{n}$. Denoting the crosstalk-induced variations by $X_{n}$, so that $h[n]=\langle h[n]\rangle+X_{n} \simeq P_{n+1}+X_{n}$, the rms standard deviation, averaged over the $N$ positions of the sequence, is:

$$
\bar{\sigma}_{X}=\left(\frac{1}{N} \sum_{n=0}^{N-1}\left\langle X_{n}^{2}\right\rangle\right)^{1 / 2}=\frac{2 \pi}{\sqrt{3}} \sigma \bar{P}
$$

with $\bar{P}=\left(N^{-1} \sum_{n=1}^{N} P_{n}^{2}\right)^{1 / 2}$ the rms power reflected by the elements of the array. This formula, derived in the appendix, predicts that, for the standard deviation $\sigma=0.016$ found in our experiments, crosstalk can reach a level of $-12 \mathrm{~dB}$ below the rms power level. This value of $\sigma$ is representative of 


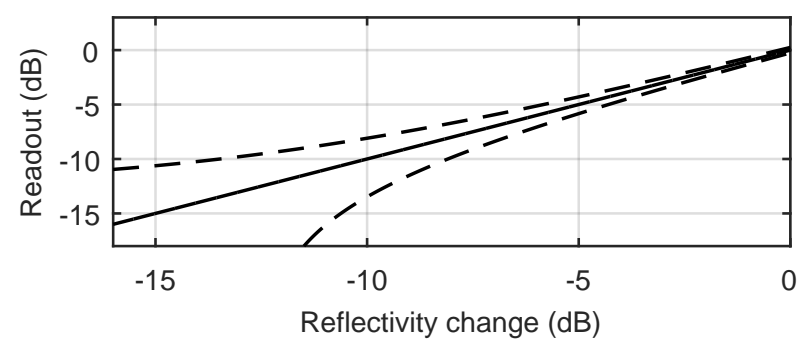

Fig. 2. Ideal readout (continuous trace) and readout bounds (dashed traces) of reflectivity changes $\Delta R=10 \log _{10} \ell$ in an array with $\sigma=0.016$ and $N=10$.

the positioning error in commercial FBG arrays built in draw towers, which typically show a maximum deviation $<5 \%$ [27].

This crosstalk induces deviations in the readout of reflectivities, which are higher at larger background rms powers relative to the measured reflectivity. Let us assume that initially all FBGs reflect the same power $P$, so that $\bar{P}=P$. If the reflectivity of one of the FBGs decreases by a factor $\ell \leq 1$, the expected readout range is $\ell P \pm \bar{\sigma}_{X}$. This range is depicted in Fig. 2 with dashed traces as a function of the reflectivity change $\Delta R=10 \log _{10} \ell$ for $N=10$. At $\Delta R=0 \mathrm{~dB}(\ell=1$, initial state), the readout may vary by $\pm 0.3 \mathrm{~dB}$, whereas at $\Delta R=-10 \mathrm{~dB}$ the measured value may lie in the range between $-8 \mathrm{~dB}$ and $-14 \mathrm{~dB}$. Therefore, crosstalk limits the dynamic range of accurate reflectivity measurements, which in Fig. 2 is $\Delta R \simeq-6 \mathrm{~dB}$ for an accuracy of $\pm 1 \mathrm{~dB}$, reflecting the basic compromise underlying the proposed technique.

\section{Phaseless Interrogation}

In contrast to the vector mode, the phaseless mode retrieves the $P_{n}$ values only from RF power responses after a modification of the array structure and the interrogation frequencies. First, a reference FBG with reflected power $P_{0}$ is introduced at a location $\Delta l / 2$ before the first FBG in the array, as shown in Fig. 1(b). The transfer function is given by:

$$
H^{\prime}(f)=P_{0}+\exp \left(-j 2 \pi f \frac{\Delta \tau}{2}\right) H(f),
$$

with $H(f)$ given by (1). Alternatively, (8) can be understood as the response of an equivalent set of $4 N-1$ equally-spaced reflectors separated by $\Delta l / 2$, of which only those corresponding to the $N$ FBGs in the array, together with the reference reflector, are non-zero. Since the tap interval is halved in comparison with the vector mode, the periodicity in frequency of the transfer function is doubled: $F S R^{\prime}=1 /(\Delta \tau / 2)=$ $2 F S R$. This also means that the required bandwidth is doubled with respect to the vector mode. Second, the set of $4 N-1$ interrogation frequencies is now given by:

$$
f_{k}=\frac{k}{4 N-1} F S R^{\prime} \quad \text { with } k=0,1, \ldots, 4 N-2,
$$

The discrete transfer function can be written as:

$$
H^{\prime}[k]=\sum_{n=0}^{4 N-2} Q_{n} \exp \left(-j 2 \pi \frac{k n}{4 N-1}\right),
$$

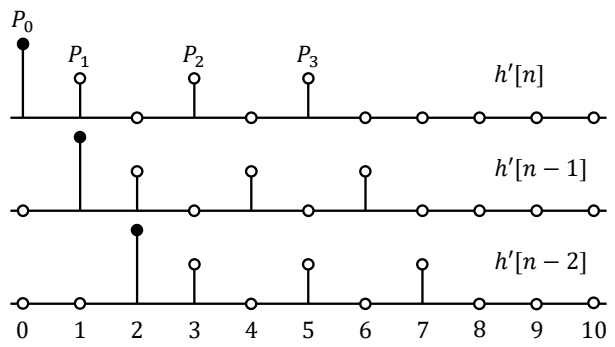

Fig. 3. Discrete-time impulse response $h^{\prime}[n]$ of a $N=3$ array with reference FBG in phaseless mode (top), together with the first two circular shifts involved in the autocorrelation. Black pins represent the reference FBG. At odd shifts $n, R[n]$ is the product of $P_{0}$ with $P_{(n+1) / 2}$.

with:

$$
Q_{n}= \begin{cases}P_{0} & \text { if } n=0 \\ P_{(n+1) / 2} & \text { if } n=1,3,5, \ldots, 2 N-1 \\ 0 & \text { otherwise. }\end{cases}
$$

The IDFT is applied to the RF power response $\left|H^{\prime}[k]\right|^{2}$. The result of this IDFT is the circular autocorrelation of the impulse response $h^{\prime}[n]=Q_{n}$ :

$$
\begin{aligned}
R[n] & =\frac{1}{4 N-1} \sum_{k=0}^{4 N-2}\left|H^{\prime}[k]\right|^{2} e^{j 2 \pi n k /(4 N-1)} \\
& =\sum_{m=0}^{4 N-2} h^{\prime *}[m] h^{\prime}[m+n(\bmod 4 N-1)],
\end{aligned}
$$

where the asterisk represents complex conjugation [28].

The result of this circular autocorrelation is exemplified in Fig. 3. This figure shows the $h^{\prime}[n]$ sequence corresponding to the array in Fig. 1(b) and the first two circular shifts associated to the $R[1]$ and $R[2]$ components of the circular autocorrelation $R[n]$. The autocorrelation components are obtained as the sum of the pointwise multiplication of sequence $h^{\prime}[n]$ in the first row and each of its circular shifts below. The result is:

$$
\begin{gathered}
R[0]=\sum_{m=0}^{N} P_{m}^{2}, \\
R[2 n-1]=R[4 N-2 n]=P_{0} P_{n}, \\
R[2 n]=R[4 N-2 n-1]=\sum_{m=1}^{N-n} P_{m} P_{m+n},
\end{gathered}
$$

with $n$ running from 1 to $N$ in (14), and from 1 to $N-1$ in (15). The $P_{n}$ values can thus be recovered from (14), whereas (15) is the (non-circular) autocorrelation of the sequence describing the FBG reflected powers. It should be noticed that in (14) the reference's power $P_{0}$ plays the role of an amplifying factor, providing selective gain to those samples in $R[n]$ used in the sensor demodulation.

As for the impact of errors in the reflectors' positions, a full statistical study is out of the scope of the present investigation. We restrict our analysis to two general observations. On the one hand, the expected performance of the phaseless mode is, in general, worse than in vector interrogation. This is because of the structure of the circular autocorrelation (12), where the 


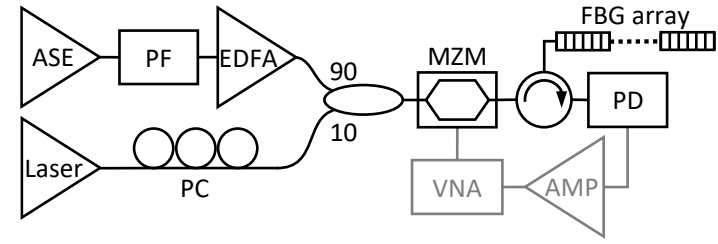

Fig. 4. Experimental setup. Black: optical path. Gray: RF path. ASE: amplified spontaneous emission source. PF: programmable filter. EDFA: erbiumdoped fiber amplifier. PC: polarization controller. MZM: Mach-Zehnder modulator. PD: photodiode. VNA: vector network analyzer. AMP: RF amplifier.

value of a particular sample $R[n]$ depends on the aggregate of products $h^{\prime *}[m] h^{\prime}[m+n]$ for all values of $m$. Variations in $h^{\prime}[m]$ due to positioning errors add in the correlation, and so the expected crosstalk level is higher. On the other hand, the limit of large reference power $P_{0}$ can be analyzed from the following representation of the discrete power response, derived from (8):

$$
\left|H^{\prime}[k]\right|^{2}=P_{0}^{2}+P_{0}\left(e^{-j 2 \pi k /(4 N-1)} H[k]+\mathrm{cc}\right)+|H[k]|^{2},
$$

where $\mathrm{cc}$ stands for complex conjugation. The IDFT of the three terms in this equation corresponds, respectively, to the $P_{0}^{2}$ contribution to the de term $R[0]$ in (13), to the two expressions in (14), and to the correlation (15). When the reference is strong, $P_{0} \gg|H[k]|$, the last term can be neglected and, apart from the constant $P_{0}^{2}$, the system simply amplifies and shifts the response $H[k]$ of the array of $N$ FBGs. In the case of strong references, we thus expect a similar behavior under crosstalk as in the vector interrogation mode.

\section{EXPERIMENTAL SETUP AND ARRAY CHARACTERIZATION}

The scheme employed in the experiment, depicted in Fig. 4, is a standard I-OFDR system based on a network analyzer (NA). The optical source combines a tunable laser (Yenista Tunics T100S-HP) emitting at the reference's Bragg wavelength, and a broadband source that feeds the rest of the FBGs in the array, so that the vector and phaseless modes can be emulated by turning the laser off and on, respectively. The broadband source is composed of an amplified spontaneous emission (ASE) source (NP Photonics C\&L Band ASE Source), filtered with a programmable amplitude and phase filter (Finisar WaveShaper 4000S), and amplified with an erbium-doped fiber amplifier (EDFA). The purpose of the filter is to equalize and emulate changes in the power reflected by the FBGs in a calibrated way. Other devices in the setup are a vector network analyzer (Agilent N5245A) operated at an IFBW of $1 \mathrm{kHz}$, unless specified; a fast photodiode $\left(\mathrm{u}^{2} \mathrm{t}\right.$ photonics XPDV2020R); and a $10-\mathrm{GHz}$ Mach-Zehnder modulator (MZM).

The array is composed of $N=10$ FBGs plus a reference FBG, all of them with different Bragg wavelengths. Its reflectivity spectrum is plotted in Fig. 5, left, where the notation follows that in Fig. 1. The FBGs have typical FWHM of 0.2 $\mathrm{nm}$ and reflectivity values $\sim-12 \mathrm{~dB}$, and were equalized by the programmable filter within $\pm 0.4 \mathrm{~dB}$. The first two FBGs
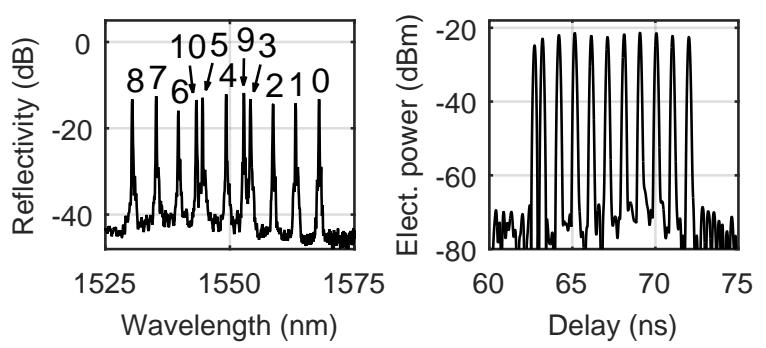

Fig. 5. Left: Array reflectivity spectrum. The numbers identify the FBGs and follow the notation of Fig. 1. Right: I-OFDR trace of the FBG array.

were spaced by $\Delta l / 2=5 \mathrm{~cm}$, whereas the rest show a mutual separation of $\Delta l=10 \mathrm{~cm}$. The resulting round-trip times were determined by I-OFDR [24]. The reflectometric trace, plotted in Fig. 5, right, showed a round-trip time between the first two FBGs of $0.480 \mathrm{~ns}$ and, for the rest of the array, an average round-trip time between consecutive FBGs of $0.979 \mathrm{~ns}$, with a relative standard deviation $\sigma=0.016$.

\section{INTERROGATION}

In a series of experiments, we measured the power reflected by the FBG array using both vector and phaseless modes. In the default state of the sensor array, that composed of $N=10$ FBGs, all of the elements reflect the same power. This situation will be referred to as the all-ON state of the sensor. Using the programmable filter, we simulated a decrease in the reflectivity of one or several FBGs by attenuating its illumination up to $10 \mathrm{~dB}$, slightly above the expected crosstalk level of $-12 \mathrm{~dB}$. In this case, the FBGs will be referred to as being in the OFF state. The objective is the determination of the FBGs in OFF state for different attenuation levels.

\section{A. Vector Interrogation}

In this mode, only the $N=10$ equally-spaced FBGs are illuminated by the equalized ASE source. Fig. 6 shows the RF power response, $10 \log _{10}|H(f)|^{2}$, and with circles its values at the sparse interrogation frequencies (2) that conform the $H[k]$ sequence. $H[0]$ is replaced by its equivalent at $f=F S R=1.02 \mathrm{GHz}$. In the first situation, shown in Fig. 6(a), the FBGs have been equalized to the all-ON state, whereas in Fig. 6(b) FBG \#4 has been set to the OFF state by an attenuation of $10 \mathrm{~dB}$. In the first case, the response at the interrogation frequencies follows the expected behavior, which corresponds to the DFT of a constant sequence, i. e., to a high dc level followed by $N-1$ zeroes. In the second plot, we observe the modification in the sampled amplitudes originated by the sole attenuation of FBG \#4.

The reflectivity readouts are shown with black and dark gray bars in Fig. 7(a). These readouts represent the normalized amplitudes of the impulse response sequences, $|h[n]|$, computed by the IDFT from the complete vector response. The third set, in light gray bars, is the result of setting FBGs \#2, 4, 6,8 and 10 to the 10-dB OFF state. These bars describe the relative attenuation of each FBG reflectivity with respect to the average value when all of them are in ON state. In the first 


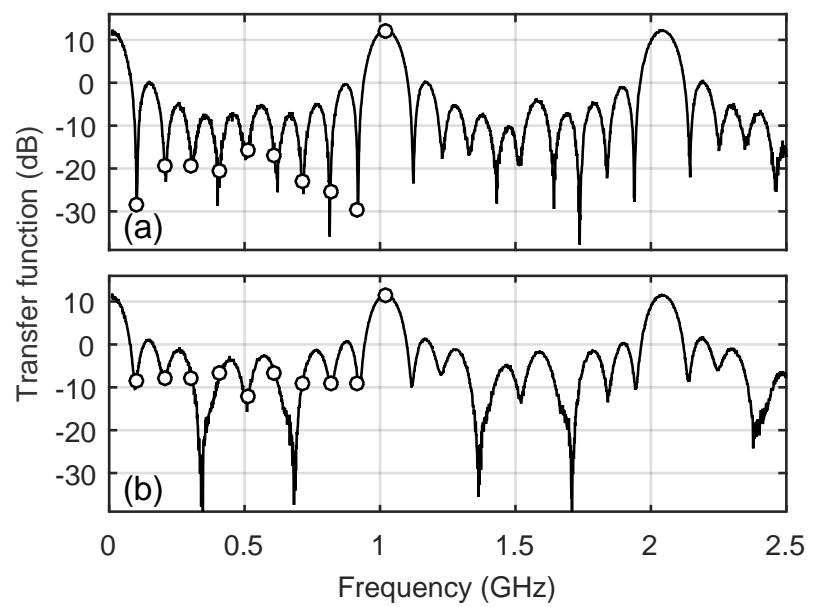

Fig. 6. System's power responses (traces) and interrogation frequencies (circles), for the vector interrogation mode when (a) the array is in the all-ON state, and (b) when the FBG \#4 is in OFF state. The traces were normalized by the response of the isolated first grating to set the phase reference to the first reflector.

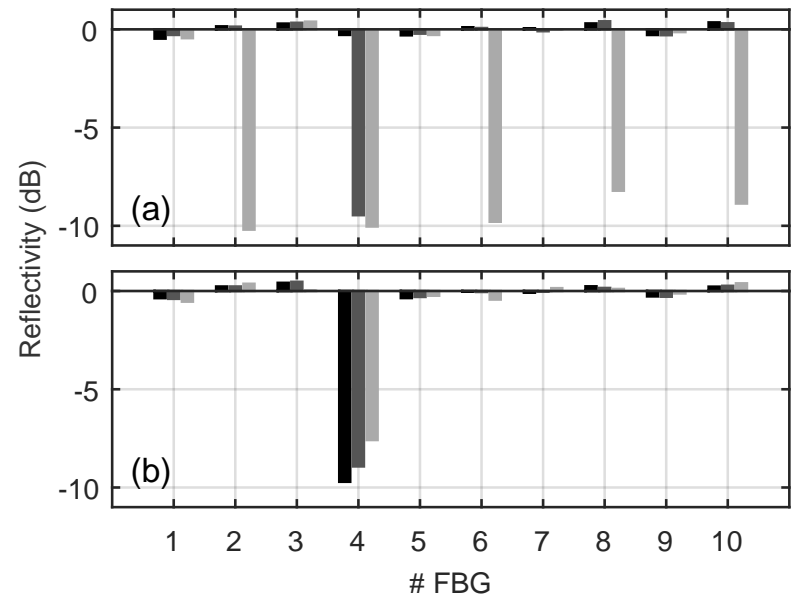

Fig. 7. Reflectivity readouts in vector interrogation mode, at IFBW $1 \mathrm{kHz}$. (a) All-ON state (black); FBG \#4 in OFF state (dark gray); and FBGs \#2, 4 , 6,8 , and 10 in OFF state (light gray). (b) FBG \#4 in OFF state with IFBWs: $1 \mathrm{~Hz}$ (black), $10 \mathrm{kHz}$ (dark gray), and $100 \mathrm{kHz}$ (light gray). The readouts were normalized relative to a $0 \mathrm{~dB}$ level defined by the mean value of the reflectivity in the all-ON state.

example (Fig. 7(a), black bars), the standard deviation of the all-ON state is $\pm 0.3 \mathrm{~dB}$, of the order of our equalization $( \pm 0.4$ $\mathrm{dB})$ and the theoretically computed deviation due to crosstalk $( \pm 0.3 \mathrm{~dB})$. When only the reflection of FBG \#4 is attenuated (Fig. 7(a), dark gray bars), the detected decay is $-9.5 \mathrm{~dB}$, close to the exact value. As expected, when more FBGs are set to the OFF state, the accuracy in the decay determination decreases due to the local variation of the crosstalk level, as it is observed in the measurements for FBGs \#8 and 10. Nevertheless, these decays are still well resolved. In a series of experiments, we attenuated by $10 \mathrm{~dB}$ several combinations of up to five gratings, finding that in all cases the decay readouts fall within the range $-11.5 \mathrm{~dB} /-8.0 \mathrm{~dB}$.

Subsequently, we characterized the sensor with only FBG \#4 in the 10-dB OFF state at increasing values of the NA's

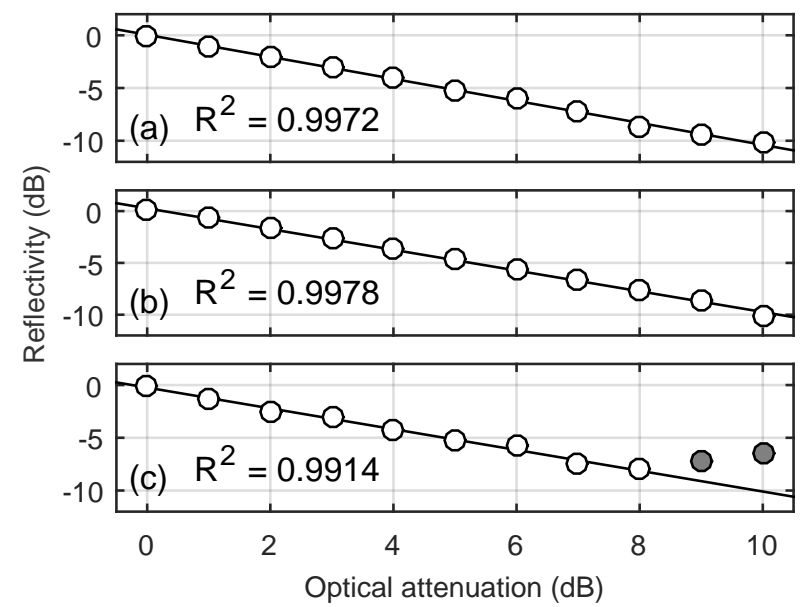

Fig. 8. Readout of the relative power reflected by FBG \#6 as a function of the attenuation when the rest of FBGs are in the ON state. Readouts from (a) the impulse response sample $h[6]$ in vector interrogation, and the circular autocorrelation sample $R[11]$ in phaseless interrogation with (b) and without (c) correlation gain. Lines represent the linear fits of the eleven data points, except in (c), where the last two measurements, in gray, were discarded. The $R^{2}$ values stand for the coefficients of determination of the linear fits.

IFBW, from $1 \mathrm{~Hz}$ to $100 \mathrm{kHz}$. Representative results are shown in Fig. 7(b). In the range $1 \mathrm{~Hz}-1 \mathrm{kHz}$ the readouts are almost invariant (within $\pm 0.1 \mathrm{~dB}$ ), indicating that the noise level is still well below the readout level. At $10 \mathrm{kHz}$ and $100 \mathrm{kHz}$, the readouts of FBG \#4 increase by $0.8 \mathrm{~dB}$ and 2.1 $\mathrm{dB}$, respectively, so that the noise level becomes progressively closer to the induced attenuation. Further increase in IFBW above $100 \mathrm{kHz}$ would lead to measurements limited by noise, not by crosstalk. At our faster scan at IFBW $100 \mathrm{kHz}$, the total scan time was $100 \mu \mathrm{s}$, which amounts to a relative scan time of $10 \mu$ s per trace point or per FBG.

We finally performed another set of measurements, consisting in reducing the FBG \#6 illumination power in 1-dB steps down to $10 \mathrm{~dB}$ at our default value of $1 \mathrm{kHz}$ IFBW, while keeping the rest of FBGs in the ON state. The results are presented in Fig. 8(a), showing that the readout linearity is maintained. According to the previous discussions, the readouts, and thus their linearity, can be impacted by a change in the background, and also by noise through the increase in IFBW, with these effects becoming lower as the values of the induced attenuation decrease.

\section{B. Phaseless Interrogation}

In a first experiment, the laser was set so that the reflected optical power on the reference was $\sim 9 \mathrm{~dB}$ higher than the average optical power reflected by the rest of FBGs, in order to provide gain to the measurement samples in the circular autocorrelation. Fig. 9(a) and (b) show, respectively, the transfer function amplitude $\left|H^{\prime}(f)\right|$ and the $\left|H^{\prime}[k]\right|$ sequence, in the all-ON state and when FBG \#4 is set to the 10-dB OFF state, in a $F S R^{\prime}=2.04 \mathrm{GHz}$.

Fig. 10(a) depicts the relative change in reflectivity measured from the amplitude of the first $N$ odd coefficients of the circular autocorrelation $R[n]$. There, the all-ON state is shown again with black bars, and with dark gray bars for 


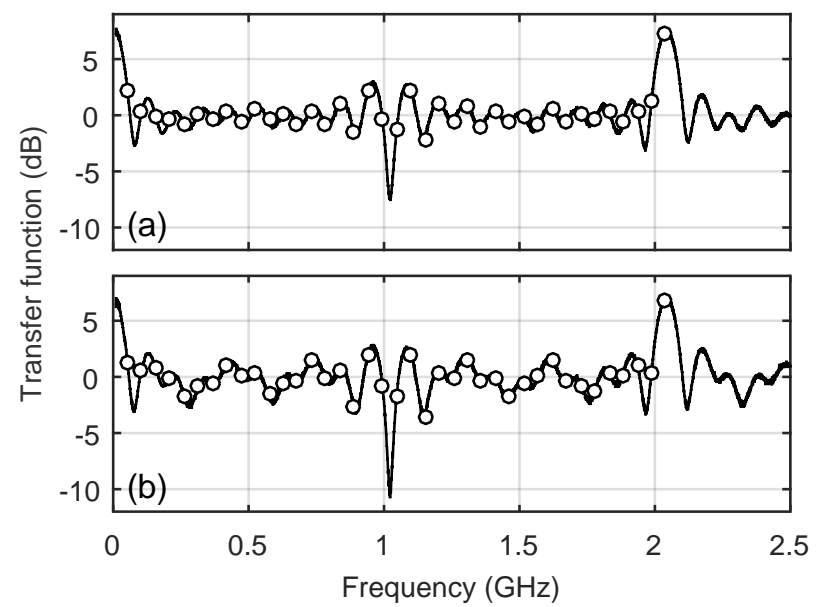

Fig. 9. System's power responses (traces) and interrogation frequencies (circles), for the phaseless interrogation mode with correlation gain when (a) the array is in the all-ON state, and (b) when the FBG \#4 is in OFF state. The traces were normalized by the response of the isolated first grating.

\#4 OFF. The third case where FBGs \#2, 4, 6, 8, and 10 are OFF is also shown with light gray bars. The all-ON state presents a deviation of $\pm 0.5 \mathrm{~dB}$, comparable to that obtained in the vector interrogation mode, and the attenuation value found for the isolated FBG \#4 is $-9.7 \mathrm{~dB}$. We repeated the same attenuation test for several groups of up to five gratings, obtaining measured decays in the range $-12.1 \mathrm{~dB} /-6.9 \mathrm{~dB}$. Despite the fact that deviations are larger than in vector mode, reflectivity decays can still be unambiguously detected. We also explored the readout linearity by attenuating the FBG \#6. The results, plotted in Fig. 8(b), indicate a good linearity, similar to that found in the vector mode. Overall, the performance shown is similar to the vector mode, a fact that can be attributed, as explained in Section III, to the presence of a high reference level that provides selective gain in the readout samples. The scanning time, however, increases by a factor $(4 N-1) / N \sim 4$ with respect to vector mode at any value of IFBW, due to the increase in the number of interrogation frequencies.

In a second series of experiments, we repeated the same tests in phaseless mode without correlation gain, using a lower reference level, $0.5 \mathrm{~dB}$ below the average power reflected by $N=10$ sensing FBGs. As shown in Fig. 10(b), the uncertainty in the readouts is higher than in the previous experiments: the standard deviation of the all-ON state is $\pm 1.6 \mathrm{~dB}$, the readout for the isolated FBG \#4 is $-9.7 \mathrm{~dB}$. The measurement range of 10-dB OFF decays found in several independent tests was $-15.7 \mathrm{~dB} /-6.8 \mathrm{~dB}$. Also, the results of the progressive attenuation test over FBG \#6, plotted in Fig. 8(c), show that linearity is only assured until an attenuation level of $8 \mathrm{~dB}$. The loss in performance found in this interrogation mode is compatible with the expected higher crosstalk level in the absence of a strong reference.

\section{CONCLUSions}

We have presented two implementations, vector and phaseless, of a sparse, low-bandwidth I-OFDR system, for the fast

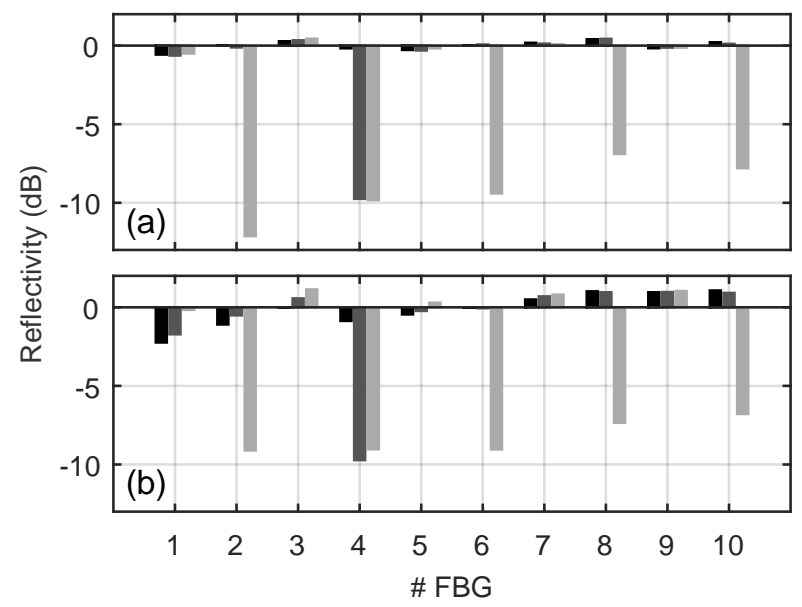

Fig. 10. Reflectivity readouts in phaseless interrogation mode with (a) and without (b) correlation gain, corresponding to an array in the all-ON state (black); FBG \#4 in OFF state (dark gray); and FBGs \#2, 4, 6, 8, and 10 in OFF state (light gray).

interrogation of equally-spaced FBG arrays based on IDFT demodulation. Dynamic range and reflectivity accuracy have been shown to be limited by the crosstalk level induced by random positioning errors in the reflectors. The two implementations have succeeded in the fast detection of 10-dB FBGs reflectivity decays, with scan times in vector mode as low as $10 \mu$ s per FBG. In phaseless mode, which in contrast to the vector mode only involves the determination of the RF power response, the scan time is increased by a factor of four due to the increase in interrogation frequencies, and reflectivity measurements worsens slightly when the implementation incorporates correlation gain, and more notably without it. Additional measurements have also shown that readout linearity is maintained in ranges up to $10 \mathrm{~dB}$, thus permitting the definition of different decay thresholds. Understood as discrete I-OFDR systems, the proposed techniques point to new routes to trade interrogation time with dynamic range and accuracy in reflectivity measurements, through the number and values of interrogation frequencies and the sensor topology, which can be applied to arrays of broad or narrowband reflectors, in the latter case at equal or different wavelengths, or integrated in systems incorporating wavelength scan.

\section{APPENDIX}

Using Parseval's theorem, the variance, averaged over the $N$ samples, only depends on the average variance in the frequency domain:

$$
\begin{aligned}
\bar{\sigma}_{X}^{2} & =\frac{1}{N} \sum_{n=0}^{N-1} \sigma_{X n}^{2}=\frac{1}{N} \sum_{n=0}^{N-1}\left(\left\langle|h[n]|^{2}\right\rangle-|\langle h[n]\rangle|^{2}\right) \\
& =\frac{1}{N^{2}} \sum_{k=0}^{N-1}\left(\left\langle|H[k]|^{2}\right\rangle-|\langle H[k]\rangle|^{2}\right) .
\end{aligned}
$$

This quantity can be computed from (4), using that positioning errors are assumed Gaussian, independent, and identically 
distributed. In terms of the characteristic function we get:

$$
\begin{aligned}
\left\langle|H[k]|^{2}\right\rangle & -|\langle H[k]\rangle|^{2} \\
& =\sum_{n=1}^{N} P_{n}^{2} \times\left(\Phi(0)-|\Phi(k / N)|^{2}\right) .
\end{aligned}
$$

The sum over $k$ is calculated in the large $N$ limit, as it becomes a Riemann integral, from which one can extract the leading contribution of order $\sigma^{2}$. Using the definition of $\bar{P}$, we have:

$$
\begin{aligned}
\bar{\sigma}_{X}^{2} & =\frac{\bar{P}^{2}}{N} \sum_{k=0}^{N-1}\left(\Phi(0)-|\Phi(k / N)|^{2}\right) \\
& \simeq \bar{P}^{2} \int_{0}^{1} d x\left(1-e^{-4 \pi^{2} \sigma^{2} x^{2}}\right) \simeq \frac{4 \pi^{2} \sigma^{2}}{3} \bar{P}^{2}
\end{aligned}
$$

which directly gives (7). The computation is thus exact up to orders $\sigma^{2} / N$ and $\sigma^{4}$.

\section{REFERENCES}

[1] C. G. Askins, M. A. Putnam, G. M. Williams, and E. J. Friebele, "Stepped-wavelength optical-fiber Bragg grating arrays fabricated in line on a draw tower," Opt. Lett., vol. 19, no. 2, pp. 147-149, Jan. 1994.

[2] A. D. Kersey, T. A. Berkoff, and W. W. Morey, "Multiplexed fiber Bragg grating strain-sensor system with a fiber Fabry-Perot wavelength filter," Opt. Lett., vol. 18, no. 16, pp. 1370-1372, Aug. 1993.

[3] S. H. Yun, D. J. Richardson, and B. Y. Kim, "Interrogation of fiber grating sensor arrays with a wavelength-swept fiber laser," Opt. Lett., vol. 23, no. 11, pp. 843-845, June 1998.

[4] M. Fernández-Vallejo and M. López-Amo, "Optical fiber networks for remote fiber optic sensors," Sensors, vol. 12, no. 4, pp. 3929-3951, Mar. 2012.

[5] M. Froggatt, B. Childers, J. Moore, and T. Erdogan, "High density strain sensing using optical frequency domain reflectometry," Proc. SPIE, vol. 4185 , pp. 249-255, Nov. 2000.

[6] B. A. Childers, M. E. Froggatt, S. G. Allison, T. C. Moore Sr., D. A. Hare, C. F. Batten, and D. C. Jegley, "Use of 3000 Bragg grating strain sensors distributed on four 8-m optical fibers during static load tests of a composite structure," Proc. SPIE, vol. 4332, pp. 133-142, June 2001.

[7] J. Huang, X. Lan, M. Luo, and H. Xiao, "Spatially continuous distributed fiber optic sensing using optical carrier based microwave interferometry," Opt. Express, vol. 22, no. 15, pp. 18 757-18 769, July 2014.

[8] J. Hervás, C. R. Fernández-Pousa, D. Barrera, D. Pastor, S. Sales, and J. Capmany, "An interrogation technique of FBG cascade sensors using wavelength to radio-frequency delay mapping," J. Lightwave Technol., vol. 33, no. 11, pp. 2222-2227, June 2015.

[9] J. Clement, G. Torregrosa, J. Hervás, D. Barrera, S. Sales, and C. R. Fernández-Pousa, "Interrogation of a sensor array of identical weak FBGs using dispersive incoherent OFDR," IEEE Photonics Technol. Lett., vol. 28, no. 10, pp. 1154-1156, May 2016.

[10] R. Cheng, L. Xia, C. Sima, Y. Ran, J. Rohollahnejad, J. Zhou, Y. Wen, and C. Yu, "Ultra-short FBG based distributed sensing using shifted optical Gaussian filters and microwave-network analysis," Opt. Express, vol. 24, no. 3, pp. 2466-2484, Feb. 2016.

[11] S. Werzinger, S. Bergdolt, R. Engelbrecht, T. Thiel, and B. Schmauss, "Quasi-distributed fiber Bragg grating sensing using stepped incoherent optical frequency domain reflectometry," J. Lightwave Technol., vol. 34 no. 22, pp. 5270-5277, Nov. 2016.

[12] S. Werzinger, M. Gottinger, S. Gussner, S. Bergdolt, R. Engelbrecht, and B. Schmauss, "Model-based compressed sensing of fiber Bragg grating arrays in the frequency domain," Proc. SPIE, vol. 10323, no. 6H, Apr. 2017.

[13] M. A. Putnam, M. L. Dennis, I. N. Duling III, C. G. Askins, and E. J. Friebele, "Broadband square-pulse operation of a passively mode-locked fiber laser for fiber Bragg grating interrogation," Opt. Lett., vol. 23, no. 2, pp. 138-140, Jan. 1998.

[14] Y. Wang, J. Gong, B. Dong, D. Y. Wang, T. J. Shillig, and A. Wang, "A large serial time-division multiplexed fiber Bragg grating sensor network," J. Lightwave Technol., vol. 30, no. 17, pp. 2751-2756, Sept. 2012.
[15] C. Hu, H. Wen, and W. Bai, "A novel interrogation system for large scale sensing network with identical ultra-weak fiber Bragg gratings," J. Lightwave Technol., vol. 32, no. 7, pp. 1406-1411, Apr. 2014.

[16] P. Han, Z. Li, L. Chen, and X. Bao, "A high-speed distributed ultra-weak FBG sensing system with high resolution," IEEE Photonics Technol. Lett., vol. 29, no. 15, pp. 1249-1252, Aug. 2017.

[17] M. L. Dennis, M. A. Putnam, J. U. Kang, T.-E. Tsai, I. N. Duling III, and E. J. Friebele, "Grating sensor array demodulation by use of a passively mode-locked fiber laser," Opt. Lett., vol. 22, no. 17, pp. 1362-1364, Sept. 1997.

[18] L. Ma, C. Ma, Y. Wang, D. Y. Wang, and A. Wang, "High-speed distributed sensing based on ultra weak FBGs and chromatic dispersion," IEEE Photonics Technol. Lett., vol. 28, no. 12, pp. 1344-1347, June 2016.

[19] C. Wang, Y. Shang, X.-H. Liu, C. Wang, H.-H. Yu, D.-S. Jiang, and G.-D. Peng, "Distributed OTDR-interferometric sensing network with identical ultra-weak fiber Bragg gratings," Opt. Express, vol. 23, no. 22, pp. 29038-29046, Nov. 2015.

[20] Y. Ou, C. Zhou, L. Qian, D. Fan, C. Cheng, and H. Guo, "Largecapacity multiplexing of near-identical weak fiber Bragg gratings using frequency-shifted interferometry," Opt. Express, vol. 23, no. 24, pp. 31 484-31 495, Nov. 2015.

[21] Y. Ou, C. Zhou, L. Qian, D. Fan, C. Cheng, H. Guo, and Z. Xiong, "Large WDM FBG sensor network based on frequency-shifted interferometry," IEEE Photonics Technol. Lett., vol. 29, no. 6, pp. 535-538, Mar. 2017.

[22] J. Hervás, A. Lavinia Ricchiuti, W. Li, N. H. Zhu, C. R. FernándezPousa, S. Sales, M. Li, and J. Capmany, "Microwave Photonics for optical sensors," IEEE J. Sel. Top. Quantum Electron., vol. 23, no. 2, pp. 327-339, Mar./Apr. 2017.

[23] D. W. Dolfi, M. Nazarathy, and S. A. Newton, "5-mm-resolution optical-frequency-domain reflectometry using a coded phase-reversal modulator," Opt. Lett., vol. 13, no. 8, pp. 678-680, Aug. 1988.

[24] D. Derickson, Fiber optic test and measurement. Prentice-Hall, 1998.

[25] M. Hiebel, Fundamentals of vector network analysis, 5th ed. Rohde \& Schwarz, 2011

[26] Z. Glasser, G. Zaychik, R. Abramov, D. Gotliv, and S. Sternklar, "Phaseless incoherent optical frequency domain spectroscopy," Opt. Lett., vol. 42, no. 9, pp. 1848-1851, May 2017.

[27] FBGS International. [Online]. Available: http://www.fbgs.com/

[28] J. G. Proakis and D. G. Manolakis, Digital signal processing, 4th ed. Pearson, 2007. 